\title{
Desempenho anaeróbico e ACTN3 em crianças
}

\section{Anaerobic performance and alpha-actinin-3 in children}

\author{
R.C. Montenegro, C.R. Paz, A.N. Montenegro Neto, V.S. Araújo Filho, P.R. Fernandes, \\ J. Fernandes Filho
}

ARTIGO ORIGINAL | ORIGINAL ARTICLE

\begin{abstract}
O presente estudo teve o objetivo de comparar o desempenho anaeróbico conforme as configurações genéticas RR, RX e XX da proteína ACNT3 em crianças. Utilizou-se uma metodologia descritiva e comparativa com uma amostra de 111 rapazes praticantes de futebol, com idades entre os 7 e 12 anos. A saliva serviu para extração do DNA e identificação do polimorfismo da ACTN3. Posteriormente realizou-se o teste de corrida de 40 metros para a medida da Potência muscular e o Índice de Fadiga. Com isso, foram estabelecidos os subgrupos: Grupo RR $(n=42)$; Grupo RX $(n=58)$; Grupo XX $(n=$ 11). A distribuição normal dos dados foi identificada com o teste de Kolmogorov-Smirnov, sendo a comparação realizada por meio da ANOVA one-way com post hoc de Scheffé. Os valores dos tempos das corridas entre os três grupos foram semelhantes, não ocorrendo diferenças significativas. A maior média de Potência foi encontrada no grupo de configuração genética RR; contudo, não foram verificadas diferenças significativas na Potência muscular e no Índice de Fadiga. Assim, como não foram observadas diferenças significativas na capacidade anaeróbica entre os grupos, conclui-se que na infância não é possível identificar o desempenho para o alto rendimento, utilizando-se testes físicos. Palavras-chave: força muscular, polimorfismo genético, criança
\end{abstract}

ABSTRACT

The aim of this research was to compare the anaerobic performance according to RR, RX and XX genetic configurations of alpha-actinin-3 in children. It was a descriptive as comparative study with a sample of soccer players of 111 boys from 7 to 12 years of age. DNA extraction from saliva was used for alpha-actinin-3 poliphormism identification. After that, a 40 meters sprint test was used to determinate the Power and Fatigue Index. The sample was split in three groups: RR $(n=42)$; RX ( $n=$ 58); XX $(n=11)$. The Kolmogorov-Smirnov test was used to assess data normality. The comparison was done with ANOVA one-way with Scheffé as post-hoc test. Sprinting data from the three groups showed no significant differences. The higher mean of Power was found in RR group, but there were no significant differences in Power and Fatigue Index. Since it was found no significant differences in anaerobic performance among groups, we conclude that in childhood it is not possible to identify high performance using physical tests.

Keywords: power, genetic poliphormism, children

Submetido: 11.09.2012 | Aceite: 30.03.2013

Ramon Cunha Montenegro, Carlos Renato Paz. Federal Institute of Education, Science and Technology of Paraiba IFPB, Brasil.

Asdrúbal Nóbrega Montenegro Neto, Vanduir Soares de Araújo Filho. Physical Evaluation Laboratory - UNIPÊSANNY, Brasil.

Paula Roquetti Fernandes, José Fernandes Filho. Center of Excellence in Physical Evaluation - CEAF/RJ/BR; LABIMH-UFRJ/RJ/BR Research Group, Brasil.

Endereço para correspondência: Ramon Cunha Montenegro, Instituto Federal de Educação, Ciência e Tecnologia da Paraíba - Núcleo Avançado de Guarabira, Coordenação de Pesquisa e Extensão, Rua José Américo de Almeida, s/n., Nordeste I, CEP: 58200000 - Guarabira, PB, Brasil

E-mail: proframon_cunha@hotmail.com 
A genética possui papel determinante na identificação dos fatores ligados às qualidades físicas e à prática desportiva, destacando-se as características da coordenação motora, força muscular e massa magra (Judson et al., 2011; Ropponen, Levalahti, Videman, Kaprio, \& Battie, 2004; Yang et al., 2011).

No período de 2006 a 2007, pesquisadores identificaram 214 características genéticas associadas ao desempenho desportivo humano (Bray et al., 2009).

Diversos estudos sugerem que através do polimorfismo da ACTN3 ( $\alpha$-actinina-3) é possível identificar se o indivíduo possui a predisposição genética para a potência muscular anaeróbica (Ahmetov et al., 2010; Druzhevskaya, Ahmetov, Astratenkova, \& Rogozkin, 2008; Moran et al., 2007; Niemi \& Majamaa, 2005; Norman et al., 2009; North et al., 1999; Papadimitriou, Papadopoulos, Kouvatsi, \& Triantaphyllidis, 2008; Roth et al., 2008; Yang et al., 2003).

Indivíduos homozigotos RR ou heterozigotos RX para a ACNT3 apresentam predisposição genética para desportos de curta duração e alta velocidade. Enquanto o genótipo mutante XX resulta na proteína não funcional, porém essa deficiência não resulta em patologia nem compromete a função muscular (Norman et al., 2009).

Muitos movimentos fundamentais para o desporto necessitam de execuções extremamente rápidas. Desta forma, o principal parâmetro observado durante os gestos desportivos não é a força, mas sim a velocidade com que a força muscular pode ser produzida. Partindo destas informações, o índice de fadiga (IFad) é bastante utilizado no intuito de medir a capacidade individual de suportar exercícios de alta intensidade sem deixar diminuir rapidamente sua potência. Com isso, o declínio desta ao longo do tempo reflete a fadiga muscular. Assim, indivíduos que conseguem tolerar esforços inúmeras vezes com maior eficácia metabólica e sem se cansar rapidamente possuem baixos índices de fadiga (Oliveira, 2010; Silva, 1999).
As pesquisas realizadas acerca da influência da ACTN3 sobre o desempenho atlético em desportos de curta duração e alta potência têm sido desenvolvidas com atletas adultos, de alto rendimento, porém não com crianças, com baixa intensidade de treinamento. Com isso, o presente estudo tem o objetivo de comparar o desempenho anaeróbico conforme as configurações genéticas $\mathrm{RR}, \mathrm{RX}$ e XX da proteína ACNT3 em crianças.

\section{MÉTODO}

Este estudo foi desenvolvido por meio de uma pesquisa descritiva, comparativa e transversal.

\section{Participantes}

A amostra foi composta por 111 indivíduos, selecionados por procedimento não probabilístico, do tipo intencional, todos do sexo masculino, que se encontravam na faixa etária de 7 a 12 anos, não aparentados, praticantes de futebol, habitantes com domicílio em cidades do Estado da Paraíba - Brasil, que se dispuseram a realizar a coleta da saliva (ACTN3) e o teste de esforço anaeróbico com a corrida de 40 metros (Thomas, Nelson, \& Silverman, 2012).

Todos os procedimentos foram estruturados conforme o International Committee of Medical Journal Editors - ICMJE e aprovados pelo Comitê de Ética e Pesquisa do Hospital Lauro Wanderley da Universidade Federal da Paraíba - CEP/HULW, protocolo 369/10, o qual cumpre os preceitos definidos na Declaração de Helsínquia.

Foram considerados aptos a fazer parte da pesquisa, crianças de 7 a 12 anos, do sexo masculino, praticantes de futebol, residentes em cidades do Estado da Paraíba. Com isso, foram excluídos do processo, os indivíduos que não obtiveram consentimento livre e esclarecido do pai ou responsável; os que não concordaram com os termos de compromisso assumidos com o pesquisador; os que tiveram algum grau de consanguinidade; os que tiveram problemas físicos que os impedia de participar nas avaliações; os que recusaram participação no estudo 
como voluntário, sem vantagem financeira e que não compareceram no local no dia da coleta dos dados.

\section{Instrumentos}

A estatura foi aferida por meio do estadiómetro Standard Sanny® - ES 2030 e a massa corporal foi medida por uma balança digital (Personal Line PL 150) da marca Filizola ${ }^{\circledR}$.

A coleta do material genético ocorreu por meio de swab estéril com cabo comprido de plástico, individual e com algodão hidrófilo em uma das extremidades. Utilizou-se o equipamento PCR em tempo real IQ5 Termal Cycler (Biorad) com uso do kit para determinação do polimorfismo R577X (Assay Id C_590093_1_Applied Biosystems) conforme o manual do fabricante.

A capacidade anaeróbica foi avaliada no campo de futebol do UNIPÊ por meio do Teste de Corrida de 40 metros. O momento de partida e de chegada foram medidos com o sistema de fotocélulas Speed Test 6.0 da CEFISE Biotecnologia Esportiva ${ }^{\circledR}$.

\section{Procedimentos}

Após a seleção, os sujeitos foram submetidos a uma única avaliação que constou da medida da estatura, da massa corporal, amostra das células da mucosa oral e mensuração do desempenho anaeróbico por meio do teste de corrida de 40 metros.

\section{Medidas Antropométricas}

Para medida da estatura o avaliado encontrava-se descalço, mantendo os calcanhares unidos, os braços relaxados e permanecendo o mais ereto possível com sua cabeça direcionada na vertical orientada no plano de Frankfurt. Já na medida da massa corporal o avaliado utilizava apenas uma sunga e, ao subir no centro da plataforma da balança, permanecia imóvel.

As medidas antropométricas foram executadas no Laboratório de Avaliação Física UNIPÊ/SANNY do curso de Educação Física do Centro Universitário de João Pessoa UNIPÊ.
Identificação da Genotipagem do resíduo 577 da ACTN3

O swab foi esfregado na mucosa jugal, em seguida foram recolocados nos respetivos invólucros de plástico e encaminhados ao Laboratório de DNA. As amostras coletadas foram submetidas à extração de DNA conforme Walsh, Metzger e Higuchi (1991).

A genotipagem foi realizada no Laboratório de DNA Forense do Instituto de Polícia Científica da Paraíba - IPC/PB/BR.

\section{Mensuração da capacidade anaeróbica}

Os voluntários realizaram um breve aquecimento de $10 \mathrm{~min}$. Posteriormente foram colocados na linha de partida, saindo de uma posição estática até atingir a distância de 40 metros em alta velocidade (sprint). Esse procedimento foi executado quatro vezes com intervalos de cinco minutos entre as tentativas para recuperação da fonte energética ATP (Svensson \& Drust, 2005).

A Potência média foi calculada por meio do produto entre a massa corporal do adolescente $(\mathrm{kg})$ e a distância percorrida $(40 \mathrm{~m})$, sendo seu resultado dividido pela média dos tempos dos quatro sprints, conforme a equação:

$$
\begin{gathered}
\text { Potência média }_{(\mathrm{kg} \cdot \mathrm{m} / \mathrm{s})}=\text { Massa corporal } \\
\operatorname{Distância~}_{(\mathrm{m})} / \text { Tempo médio }_{(\mathrm{s})}
\end{gathered}
$$

A Potência máxima foi calculada por meio do produto entre a massa corporal total do adolescente $(\mathrm{kg})$ e a distância percorrida (40 $\mathrm{m})$, sendo seu resultado dividido pelo menor tempo executado nos quatro sprints. Já a Potência mínima verificou-se pelo resultado da massa corporal do adolescente $(\mathrm{kg})$ e a distância percorrida $(40 \mathrm{~m})$, divididos pelo maior tempo executado nos quatro sprints.

O Índice de Fadiga foi identificado com a subtração da maior potência pela menor potência, divididas pela maior potência e multiplicada por 100 (Coledam et al., 2010).

A bateria de testes foi realizada no campo de futebol do curso de Educação Física do UNIPÊ. 


\section{Análise Estatística}

Para interpretação e identificação das diferenças entre os subgrupos amostrais utilizouse a estatística inferencial. Previamente, aplicou-se o teste Kolmogorov-Smirnov para a análise da curva de distribuição dos dados quantitativos. $\mathrm{Na}$ comparação das variáveis utilizou-se a análise de variância ANOVA oneway com post hoc de Scheffé para os casos que apontassem diferença significativa.

Em todos os procedimentos utilizou-se o nível de significância de $p<.05$, por meio do Programa Statistical Package for the Social Science - SPSS ${ }^{\circledR}$, versão 14.0.

\section{RESULTADOS}

Todos os dados apresentaram uma distribuição normal.

Na Tabela 1 verificou-se que o Grupo I (RR), correspondeu a $37.84 \%$ do total de avaliados, o Grupo II (RX) compôs $52.25 \%$ e o Grupo III (XX) correspondeu a 9.91\% da amostra. Observou-se também que o grupo RR teve a maior média de massa corporal e não ocorreram diferenças entre as variáveis idade $(p=.177)$ e massa corporal $(p=.433)$.

A tabela 2 demonstra os resultados referentes ao tempo médio, o menor e o maior tempo de duração dos sprints. A análise desta tabela mostra valores muito semelhantes para as variáveis de tempo entre os três grupos, não havendo diferenças significativas entre o tempo médio $(p=.210)$, tempo mínimo $(p=.762)$ e o tempo máximo $(p=.271)$.

A Tabela 3 exibe os resultados da potência e índice de fadiga entre os grupos. Nota-se que a maior média de potência foi encontrada no grupo de configuração genética RR, contudo, com base na ANOVA, não foram identificadas diferenças significativas na potência média ( $p=$ .331), na potência mínima $(p=.216)$ e na potência máxima $(p=.708)$.

Os resultados referentes ao índice de fadiga demonstraram proximidade dos valores, sendo o grupo RX o que apresentou maior queda de potência. A análise de variância ANOVA, não identificou diferenças significativas entre $\mathrm{O}$ IFad dos grupos $(p=.081)$.

\section{DISCUSSÃO}

A massa corporal pode ter influenciado favoravelmente no desempenho da potência do grupo I (RR), uma vez que a carga mobilizada está diretamente ligada à potência. De maneira inversa, o tempo gasto em cada sprint, que no grupo $\mathrm{XX}$, também foi maior, o que resultou

Tabela 1

Valor médio da idade e massa corporal dos grupos

\begin{tabular}{cccccc}
\hline \multirow{2}{*}{ Grupos } & $n$ & \multicolumn{2}{c}{ Idade } & \multicolumn{2}{c}{ Massa corporal } \\
\cline { 3 - 6 } & & $\mathrm{M} \pm \mathrm{DP}$ & Mínimo-Máximo & $\mathrm{M} \pm \mathrm{DP}$ & Mínimo-Máximo \\
\hline G I (RR) & 42 & $9.86 \pm 1.72$ & $7.00-12.00$ & $32.95 \pm 8.58$ & $19.20-49.90$ \\
G II (RX) & 58 & $9.41 \pm 1.53$ & $7.00-12.00$ & $31.60 \pm 6.57$ & $18.50-44.50$ \\
G III (XX) & 11 & $8.91 \pm 1.92$ & $7.00-12.00$ & $32.02 \pm 7.80$ & $21.60-45.20$ \\
\hline
\end{tabular}

Tabela 2

Medidas descritivas da variável tempo dos sprints

\begin{tabular}{cccccccc}
\hline \multirow{2}{*}{ Grupos } & $n$ & \multicolumn{2}{c}{ Tempo Médio } & \multicolumn{2}{c}{ Tempo Mínimo } & \multicolumn{2}{c}{ Tempo Máximo } \\
\cline { 3 - 8 } & & M \pm DP & Mín-Máx & M \pm DP & Mín-Máx & M \pm DP & Mín-Máx \\
\hline G I (RR) & 42 & $7.87 \pm 0.66$ & $6.50-8.84$ & $7.52 \pm 0.81$ & $6.28-8.44$ & $8.45 \pm 1.19$ & $6.50-12.60$ \\
G II (RX) & 58 & $8.14 \pm 1.18$ & $6.68-11.67$ & $7.64 \pm 1.14$ & $5.59-10.97$ & $8.85 \pm 1.33$ & $7.12-12.75$ \\
G III (XX) & 11 & $8.36 \pm 0.77$ & $6.83-9.36$ & $7.73 \pm 0.88$ & $6.50-8.78$ & $8.90 \pm 1.10$ & $7.10-10.79$ \\
\hline \multicolumn{2}{l}{ Nota: Mín - valor mínimo, Máx - valor máximo }
\end{tabular}


Tabela 3

Medidas descritivas das variáveis potência e índice de fadiga

\begin{tabular}{ccccccc}
\hline \multirow{2}{*}{ Variáveis } & \multicolumn{2}{c}{ Grupo I - RR $(\mathrm{n}=42)$} & \multicolumn{2}{c}{ Grupo II $-\mathrm{RX}(\mathrm{n}=58)$} & \multicolumn{2}{c}{ Grupo III - XX $(\mathrm{n}=11)$} \\
& $\mathrm{M} \pm \mathrm{DP}$ & Mín-Máx & $\mathrm{M} \pm \mathrm{DP}$ & Mín-Máx & $\mathrm{M} \pm \mathrm{DP}$ & Mín-Máx \\
\hline PMéd & $115.26 \pm 46.94$ & $47.46-203.88$ & $106.27 \pm 46.37$ & $20.94-226.31$ & $93.23 \pm 42.22$ & $52.94-195.27$ \\
PMín & $95.70 \pm 43.45$ & $33.99-194.01$ & $82.72 \pm 35.96$ & $18.22-168.32$ & $79.80 \pm 39.71$ & $34.64-173.45$ \\
PMáx & $137.42 \pm 66.99$ & $51.83-270.66$ & $130.99 \pm 64.79$ & $25.21-288.53$ & $119.91 \pm 52.15$ & $63.77-201.86$ \\
IFad & $24.32 \pm 20.16$ & $0.73-87.44$ & $33.10 \pm 17.77$ & $0.64-64.57$ & $30.25 \pm 22.33$ & $1.81-70.11$ \\
\hline
\end{tabular}

Nota: Mín - valor mínimo, Máx - valor máximo; Pot $_{(\mathrm{kg} . \mathrm{m} / \mathrm{s})}$ - Potência; PMáx ${ }_{(\mathrm{kg} . \mathrm{m} / \mathrm{s})}$ - Potência Máxima; PMín $(\mathrm{kg.m} / \mathrm{s})$ -

Potência Mínima; IFad (\%) - Índice de Fadiga

em menor potência.

Diversos estudos têm mostrado que existe associação entre a performance de atletas de elite e os diferentes genótipos da ACTN3 (Ahmetov et al., 2010; Druzhevskaya et al., 2008; Eynon et al., 2009; Moran et al., 2007; Niemi \& Majamaa, 2005; Papadimitriou et al., 2008; Roth et al., 2008; Scott et al., 2010).

$\mathrm{O}$ presente estudo não reportou diferenças significativas da potência anaeróbia e do índice de fadiga entre os grupos de crianças com configuração genética RR, RX e XX. Vale ressaltar que nenhum desses estudos anteriores comparou a performance física com o polimorfismo da ACTN3 em crianças.

Num estudo realizado com 512 adolescentes gregos do sexo masculino, com idade entre os 11 e 18 anos, classificados como: 124 inativos, 157 levemente ativos, 97 ativos intermediários e 134 altamente ativos (Moran et al., 2007) verificou-se uma associação significativa $(p=0.003)$ entre os polimorfismos R577X ACTN3 e o tempo de corrida de $40 \mathrm{~m}$. Os genótipos RR e RX que representaram 34\% e $48 \%$ da amostra, respetivamente, apresentaram maior força explosiva executando a corrida de $40 \mathrm{~m}$ em menos tempo do que os adolescentes que tinham a configuração XX.

A frequência do genótipo XX significativamente menor em atletas de elite de sprints sugere que a ausência da proteína ACTN3 ativa, expressa pelo genótipo XX pode prejudicar o desempenho da potência anaeróbica e reforça que o alelo R do gene ACTN3 é um fator importante para o desempenho de um alto nível de potência nesses atletas (Druzhevskaya et al., 2008; Eynon et al., 2009; Niemi \& Majamaa, 2005; Papadimitriou et al., 2008; Roth et al., 2008; Yang et al., 2005).

No presente estudo não foram encontradas diferenças significativas da potência anaeróbica e do índice de fadiga com a configuração genética da ACTN3 das crianças avaliadas. A investigação genética sobre o fenótipo pode ser mais eficaz em crianças, pelo fato de terem sofrido menos influência externa do que os adultos (Moran et al., 2007). Os avaliados do presente estudo não foram classificados como atletas de elite como nas pesquisas já realizadas, isso é relevante, pois o desenvolvimento físico pode ser influenciado potencialmente pelo treinamento e outros fatores ambientais.

A genética tem grandes implicações sobre a otimização do desempenho atlético (Dias, Pereira, Negrão, \& Krieger, 2007). Porém, somente em algumas situações, como nos desportos de alto rendimento, é que os indivíduos com configuração RR ou RX para ACTN3, terão vantagem sobre os que não possuem a proteína ativa, uma vez que a predisposição genética aliada a fatores ambientais poderá contribuir de forma significativa para um melhor desempenho em atividades que exijam melhor força explosiva (Weinberger et al., 1996).

Nossos resultados mostraram uma menor frequência para homozigotos XX. De acordo com North et al. (1999), o genótipo XX para 
ACTN3, pode ocorrer em $16 \%$ da população não incluída na categoria atletas de elite. Em outro estudo verificou-se que não houve aumento da frequência do genótipo XX nos atletas de endurance, sugerindo que a ausência de ACTN3 ativa não influenciou o desempenho dos atletas africanos (Yang et al., 2007).

Quanto ao índice de fadiga, o grupo RR apresentou menor valor, ou seja, possuíam maior capacidade de realizar exercício máximo sem deixar cair rapidamente sua potência, esses dados corroboram estudos que sugerem que o genótipo RR seja vantajoso para o treinamento de endurance, vez que induz ao armazenamento de energia ou o uso mais eficiente desta. Porém, esse menor índice pode estar relacionado com uma potência de pico relativamente mais baixa, sem queda acentuada desta, já que quanto mais lenta for à diminuição da potência de pico, mais baixo será o índice de fadiga (MacArthur \& North, 2004; Moran et al., 2007).

O genótipo ACTN3 está associado à velocidade e potência muscular dos atletas europeus, mas não foram encontradas associações entre as medidas objetivas da capacidade física na população em geral (Alfred et al., 2011).

\section{CONCLUSÕES}

Dado que não foram observadas diferenças significativas na capacidade anaeróbica e índice de fadiga entre os grupos, conclui-se que na infância, não é possível identificar o desempenho para o alto rendimento, utilizando-se testes físicos.

Agradecimentos:

Nada a declarar.

\section{Conflito de Interesses:}

Nada a declarar.

Financiamento:

Nada a declarar.

\section{REFERÊNCIAS}

Ahmetov, I., Druzhevskaya, A. M., Astratenkova, I. V., Popov, D. V., Vinogradova, O. L., \& Rogozkin, V. A. (2010). The ACTN3 R577X polymorphism in Russian endurance athletes. British Journal of Sports Medicine, 44(9), 649-652. doi: 10.1136/bjsm.2008.051540

Alfred, T., Ben-Shlomo, Y., Cooper, R., Hardy, R., Cooper, C., Deary, I. J., \& Day, I. N. (2011). ACTN3 genotype, athletic status, and life course physical capability: Meta-analysis of the published literature and findings from nine studies. Human Mutation, 32(9), 1008-1018. doi: 10.1002/humu.21526

Bray, M. S., Hagberg, J. M., Perusse, L., Rankinen, T., Roth, S. M., Wolfarth, B., \& Bouchard, C. (2009). The human gene map for performance and health-related fitness phenotypes: The 2006-2007 update. Medicine and Science in Sports and Exercise, 41 (1), 35-73.

Coledam, D. H., Santos, D., \& Santos, J. W. (2010). Avaliação da potência anaeróbia antes e após o período competitivo em atletas profissionais de futebol. Conexões (Campinas), 2(8), 93-102.

Dias, R., Pereira, A. C., Negrão, C. E., \& Krieger, J. (2007). Genetic polymorphisms determining of the physical performance in elite athletes. Revista Brasileira de Medicina do Esporte, 13(3), 209-216.

Druzhevskaya, A. M., Ahmetov, I., Astratenkova, I. V., \& Rogozkin, V. A. (2008). Association of the ACTN3 R577X polymorphism with power athlete status in Russians. European Journal of Applied Physiology, 103(6), 631-634. doi: 10.1007/s00421-008-0763-1

Eynon, N., Alves, A. J., Yamin, C., Sagiv, M., Duarte, J. A., ... Meckel, Y. (2009). Is there an ACE ID ACTN3 R577X polymorphisms interaction that influences sprint performance? International Journal of Sports Medicine, 30(12), 888-891. doi: 10.1055/s-0029-1238291

Judson, R. N., Wackerhage, H., Hughes, A., Mavroeidi, A., Barr, R. J., Macdonald, H. M., \& Hocking, L. J. (2011). The functional ACTN3 577X variant increases the risk of falling in older females: Results from two large independent cohort studies. Journals of Gerontology Series A: Biological Sciences and Medical Sciences, 66(1), 130-135. doi: 10.1093/gerona/glq189

MacArthur, D., \& North, K. (2004). A gene for speed? The evolution and function of alphaactinin-3. BioEssays, 26(7), 786-795. 
Moran, C., Yang, N., Bailey, M., Tsiokanos, A., Jamurtas, A., MacArthur, D., Wilson, R. (2007). Association analysis of the ACTN3 R577X polymorphism and complex quantitative body composition and performance phenotypes in adolescent Greeks. European Journal of Human Genetics, 15, 88-93. doi:10.1038/sj.ejhg.5201724

Niemi, A. K., \& Majamaa, K. (2005). Mitochondrial DNA and ACTN3 genotypes in Finnish elite endurance and sprint athletes. European Journal of Human Genetics, 13, 965-969. doi: 10.1038/ sj.ejhg.5201438

Norman, B., Esbjornsson, M., Rundqvist, H., Osterlund, T., von Walden, F., \& Tesch, P. A. (2009). Strength, power, fiber types, and mRNA expression in trained men and women with different ACTN3 R577X genotypes. Journal of Applied Physiology, 106(3), 959-965. doi: 10.1152/japplphysiol.91435.2008

North, K. N., Yang, N., Wattanasirichaigoon, D., Mills, M., Easteal, S., \& Beggs, A. H. (1999). A common nonsense mutation results in alphaactinin-3 deficiency in the general population. Nature Genetics, 21, 353-354. doi: 10.1038/7675

Oliveira, A. R., Corrêa, F. I., Valim, M. M., Oliveira, C. S., \& Corrêa, J. C. F. (2010). Determination of muscle fatigue index for strength training in patients with Duchenne dystrophy. Fisioterapia em Movimento, 23(3), 351-360.

Papadimitriou, I. D., Papadopoulos, C., Kouvatsi, A., \& Triantaphyllidis, C. (2008). The ACTN3 gene in elite Greek track and field athletes. International Journal of Sports Medicine, 29(4), 352355. doi: 10.1055/s-2007-965339

Ropponen, A., Levalahti, E., Videman, T., Kaprio, J., \& Battie, M. C. (2004). The role of genetics and environment in lifting force and isometric trunk extensor endurance. Physical Therapy, 84(7), 608-621.

Roth, S. M., Walsh, S., Liu, D., Metter, E., Ferrucci, L., \& Hurley, B. F. (2008). The ACTN3 R577X nonsense allele is under-represented in elitelevel strength athletes. European Journal of $\mathrm{Hu}$ man Genetics, 16(3), 391-394. doi: 10.1038/sj. ejhg.5201964

Scott, R., Irving, R., Irwin, L., Morrison, E., Charlton, V., Austin, K., \& Pitsiladis, Y. P. (2010).
ACTN3 and ACE genotypes in elite Jamaican and US sprinters. Medicine and Science in Sports and Exercise, 42(1), 107-112. doi: 10.1249/MSS. 0b013e3181ae2bc0

Silva, P., Roxo, C., Visconti, A., Teixeira, A., Rosa, A., Firmino, M., ... Sousa, J. (1999). Índices de aptidão funcional em jogadores de futebol da Seleção Nacional da Jamaica. Revista Brasileira de Medicina do Esporte, 5(3), 93-98.

Svensson, M., \& Drust, B. (2005). Testing soccer players. Journal of Sports Sciences, 23(6), 601-618. doi: 10.1080/02640410400021294.

Thomas, J. R., Nelson, J. K., \& Silverman, S. J. (2012). Métodos de Pesquisa em Atividade Física (6 $6^{\mathrm{a}}$ ed.). Porto Alegre: Artmed.

Walsh, P. S., Metzger, D. A., \& Higuchi, R. (1991). Chelex 100 as a medium for simple extraction of DNA for PCR-based typing from forensic material. BioTechniques, 10(4), 506-513.

Weinberger, R., Schevzov, G., Jeffrey, P., Gordon, K., Hill, M., \& Gunning, P. (1996). The molecular composition of neuronal microfilaments is spatially and temporally regulated. The Journal of Neuroscience, 16(1), 238-252.

Yang, N., MacArthur, D., Gulbin, J., Hahn, A., Beggs, A., Easteal, S., \& North, K. (2003). ACTN3 genotype is associated with human elite athletic performance. American Journal of Human Genetics, 73(3), 627-631. doi: 10.1086/ 377590

Yang, N., MacArthur, D., Wolde, B., Onywera, V., Boit, M., Lau, S., \& North, K. (2007). The ACTN3 R577X polymorphism in East and West African athletes. Medicine and Science in Sports and Exercise, 39(11), 1985-1988. doi: 10.1249/mss.0b013e31814844c9

Yang, N., MacArthur, D., Wolde, B., Onywera, V., Boit, M., ... \& North, K. (2005). ACTN3 genotype is not associated with elite endurance athlete status in Ethiopians and Kenyans. Medicine and Science in Sports and Exercise, 37(5), S472.

Yang, N., Schindeler, A., McDonald, M., Seto, J., Houweling, P., Lek, M., \& North, K. (2011). Alpha-Actinin-3 deficiency is associated with reduced bone mass in human and mouse. Bone, 49, 790-798. doi: 10.1016/j.bone.2011.07.009.

(c) EY-NC Todo o conteúdo da revista Motricidade está licenciado sob a Creative Commons, exceto quando especificado em contrário e nos conteúdos retirados de outras fontes bibliográficas. 